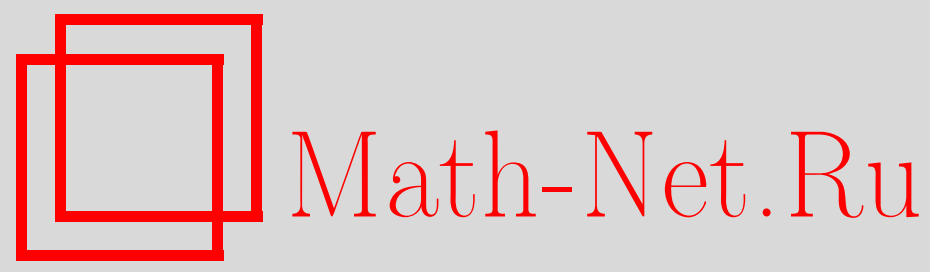

С. Тажетдинов, Субнормальное строение двумерных линейных групп над полными кольцами, Матем. заметки, 2002, том 71, выпуск 6, 924-930

DOI: https://doi.org/10.4213/mzm396

Использование Общероссийского математического портала Math-Net.Ru подразумевает, что вы прочитали и согласны с пользовательским соглашением http://www . mathnet.ru/rus/agreement

Параметры загрузки:

IP : 18.234 .197 .8

26 апреля 2023 г., 13:00:56

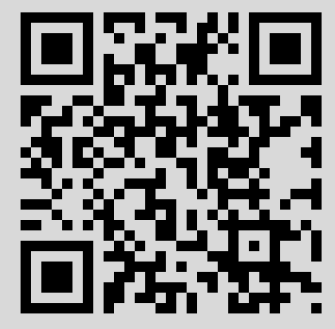




\section{СУБНОРМАЛЬНОЕ СТРОЕНИЕ ДВУМЕРНЫХ ЛИНЕЙНЫХ ГРУПП НАД ПОЛНЫМИ КОЛЬЦАМИ \\ С. Тажетдинов}

Дается описание субнормальных подгрупп двумерных линейных групп над некоторьми $(m, n)$-полньми кольцами. Примерами $(m, n)$-полных колец являются полулокальные кольца и кольца размерности 0 ; в частности, кольца, регулярные по Нейману, при условии, что каждое поле вычетов этих колец содержит более $m(n-1)$ элементов.

Библиограффия: 7 названий.

Строка матришы над кольцом $R$, элементы которой порождают как идеал все кольцо $R$, называется унимодулярной. Пусть $m \geqslant 1$ и $n \geqslant 2$ - целые числа. Согласно [1] коммутативное кольцо $R$ назьвается $(m, n)$-nолным. , если для каждой $(m \times n)$-матришы $A$ над $R$ с унимодулярными строками существуют элемент $\varepsilon \in R$ (зависяший от $A$ ) и обратимые элементы $\varepsilon_{1}, \varepsilon_{2}, \ldots, \varepsilon_{m} \in R$ такие, что

$$
A\left[1, \varepsilon, \varepsilon^{2}, \ldots, \varepsilon^{n-1}\right]^{t}=\left[\varepsilon_{1}, \varepsilon_{2}, \ldots, \varepsilon_{m}\right]^{t},
$$

где $t$ означает транспонирование. Ясно, что $(m, n)$-полное кольцо является и $(s, t)$-полным при $s \leqslant m$ и $t \leqslant n$. Таким образом, любое $(m, n)$-полное кольцо является $(1,2)$-полным кольцом, т.е. кольцом стабильного ранга 1. Известно (см. [1]), что если каждое поле вычетов полулокального кольца и кольца размерности 0 , в том числе кольца, регулярного по Нейману, содержит более $m(n-1)$ элементов, то оно является $(m, n)$-полным кольцом.

Далее, напомним, что если сушествует ряд

$$
H=H_{0} \unlhd H_{1} \unlhd \cdots \unlhd H_{d-1} \unlhd H_{d}=G
$$

подгрупп группы $G$, где $H_{i-1} \unlhd H_{i}$ означает, что $H_{i-1}$ является нормальной подгруппой $H_{i}$, то $H$ называется субнормальной подгрупой групшы $G$. В этом случае пишут $H \triangleleft^{d} G$. Наименьшее такое $d$ называется субнор.мальной глубиной $H$ в $G$.

Пусть теперь $R$ - $(1,6)$-полное, $(2,4)$-полное или $(3,3)$-полное кольцо. В настоящей работе дается описание субнормальньг подгрупп двумерных линейных групп над кольцом $R$, являющееся значительным улучшением описания из [2]. Дается также улучшение аналогичного описания для случая примитивных колец, данного в работе [3]. 
Пусть $I$ - идеал кольца $R$. Гомоморфизм колец $R \rightarrow R / I$ индуцирует сюръективньй (см. [1]) гомоморфизм групп $\pi_{I}: \mathrm{GL}_{2}(R) \rightarrow \mathrm{GL}_{2}(R / I)$. Здесь $\mathrm{GL}_{2}(R / R)$ - единичная группа. Пусть

$$
\begin{gathered}
Z_{I}=\left(\text { center } \mathrm{GL}_{2}(R / I)\right) \pi_{I}^{-1}, \quad K_{I}=\left\{\sigma \in \mathrm{SL}_{2}(R) / \sigma \pi_{I}=1\right\}, \\
E_{I}=\operatorname{gr}\left(t_{12}(\alpha), t_{21}(\alpha) \mid \alpha \in I\right),
\end{gathered}
$$

где $t_{i j}$ - трансвекции. Заметим, что $Z_{R}=\mathrm{GL}_{2}(R), Z_{0}=$ center $\mathrm{GL}_{2}(R), K_{R}=\mathrm{SL}_{2}(R)$, $K_{0}=\{1\}$. Весом матрииы

$$
h=\left(\begin{array}{ll}
a & b \\
c & d
\end{array}\right)
$$

назовем идеал $J(h)=\mathrm{id}(a-d, b, c)$, а вес подгрупnы $H$ определим формулой

$$
J(H)=\sum_{h \in H} J(h) .
$$

Группу обратимых элементов кольца $R$ обозначим через $R^{*}$.

ЛЕмма 1. Eсли подгруппа $H$ группы $\mathrm{GL}_{2}(R)$ нормализуется подгруппой $K_{I}$, то $H \geqslant K_{J}$, әде $J=2^{15} J^{2}(H) I^{7}$.

ДокАЗАТЕЛЬСТво. Подвыражением $i_{n}$ будем понимать произвольньй элемент идеала $I^{n}$. Пусть

$$
h=\left(\begin{array}{ll}
a & b \\
c & d
\end{array}\right)
$$

- матрица из $H$ и $\alpha \in I$. По определению кольца $R$ существует элемент $\varepsilon \in R$ такой, что все элементы $\varepsilon, 1+2 c \alpha \varepsilon, 1+c \alpha \varepsilon, 1+2 c \alpha \varepsilon+2 c^{2} \alpha^{2} \varepsilon^{2}$ принадлежат $R^{*}$. Если теперь $q=1+2 c \alpha \varepsilon, \nu=4 a \alpha \varepsilon(1+c \alpha \varepsilon)$ и $d(q)=\operatorname{diag}\left(q^{-1}, q\right)$, то в $H$ содержится матрица

$$
\left[h, d(q) t_{12}(\nu), t_{12}(\nu)\right]=t_{12}\left(-2^{5} c a \alpha^{2} \varepsilon^{2}\left(1+2 c \alpha \varepsilon+2 c^{2} \alpha^{2} \varepsilon^{2}\right)(1+c \alpha \varepsilon)^{2}\right),
$$

т.е.

$$
t_{12}\left(2^{5} c a \alpha^{2} \varepsilon_{1}\right) \in H,
$$

где $\varepsilon_{1}=\varepsilon^{2}\left(1+2 c \alpha \varepsilon+2 c^{2} \alpha^{2} \varepsilon^{2}\right)(1+c \alpha \varepsilon)^{2} \in R^{*}$.

Рассмотрев теперь при тех же $\alpha$ и $\varepsilon$ вместо $h$ матрицу $t_{12}(\beta) h t_{12}^{-1}(\beta)$ для произвольного $\beta \in I$, вместо (1) получим $t_{12}\left(2^{5} c(a+\beta c) \alpha^{2} \varepsilon_{1}\right) \in H$, где $\varepsilon_{1} \in R^{*}$ - тот же обратимый элемент, что и в (1). Вместе с (1) это дает $t_{12}\left(2^{5} c^{2} \alpha^{2} \beta \varepsilon_{1}\right) \in H$, и ввиду произвольности $\beta \in I$ имеем $t_{12}\left(2^{5} c^{2} \alpha^{2} i_{1}\right) \in H$. Отсюда, поскольку $\alpha \in I$ также был произвольным, получим

$$
t_{12}\left(2^{6} c^{2} i_{3}\right) \in H
$$

Аналогично, заменяя в этих рассуждениях $c, a, d(q), t_{12}(\nu)$ соответственно на $b, d$, $d^{-1}(q), t_{21}(\nu)$, вместо (1) и (2) получим включения

$$
\begin{gathered}
t_{21}\left(2^{5} b d \alpha^{2} \varepsilon_{2}\right) \in H, \quad \varepsilon_{2} \in R^{*}, \\
t_{21}\left(2^{6} b^{2} i_{3}\right) \in H .
\end{gathered}
$$


Повторяя далее в точности ход рассуждений работы [2, с. 100-101], получаем также включения

$$
\begin{aligned}
& t_{12}\left(2^{15} b^{2} i_{7}\right) \in H, \\
& t_{21}\left(2^{15} c^{2} i_{7}\right) \in H .
\end{aligned}
$$

Пусть теперь

$$
h_{1}=\left(\begin{array}{ll}
a_{1} & b_{1} \\
c_{1} & d_{1}
\end{array}\right), \quad h_{2}=\left(\begin{array}{ll}
a_{2} & b_{2} \\
c_{2} & d_{2}
\end{array}\right)
$$

- матрицы из $H$. Рассмотрев вместо $h$ матрицу $h_{1} h_{2} \in H$, вместо $(4)$ имеем $t_{21}\left(2^{6}\left(a_{1} b_{2}+\right.\right.$ $\left.\left.b_{1} d_{2}\right)^{2} i_{3}\right) \in H$. Отсюда ввиду произвольности $h \in H$ при выводе включения (4) получаем

$$
t_{21}\left(2^{7} a_{1} d_{2} b_{1} b_{2} i_{3}\right) \in H
$$

Из (4) и (7) следует включение $t_{21}\left(2^{7} b_{1} b_{2} \eta i_{3}\right) \in H$, где $\eta=\operatorname{det} h_{1} \cdot \operatorname{det} h_{2} \in R^{*}, \mathrm{a}$ потому и включение

$$
t_{21}\left(2^{7} b_{1} b_{2} i_{3}\right) \in H
$$

Аналогично (с помощью (2)) получается включение

$$
t_{12}\left(2^{7} c_{1} c_{2} i_{3}\right) \in H
$$

Далее, в силу (3) получим $t_{21}\left(2^{5} b_{2} d_{2} \alpha^{2} \varepsilon_{3}\right) \in H$, где $\varepsilon_{3} \in R^{*}$. Пусть теперь $r=$ $2^{5} b_{2} d_{2} \alpha^{2} \varepsilon_{3}$. Тогда из (2) имеем

$$
t_{12}\left(2^{6} r^{2} i_{3}\right) \in H
$$

Рассмотрев теперь вместо $h$ матрицу $h_{1} t_{21}(r) \in H$, вместо (2) получим

$$
t_{12}\left(2^{6}\left(c_{1}+d_{1} r\right)^{2} i_{3}\right) \in H
$$

Отсюда ввиду (2) и (10) следует, что $t_{12}\left(2^{7} c_{1} d_{1} r i_{3}\right) \in H$, т.е. $t_{12}\left(2^{12} c_{1} d_{1} b_{2} d_{2} \alpha^{2} i_{3}\right) \in H$. Ввиду произвольности элемента $\alpha \in I$ отсюда имеем

$$
t_{12}\left(2^{13} c_{1} b_{2} d_{1} d_{2} i_{5}\right) \in H
$$

Из (4), (8) и (11) теперь следует включение $t_{12}\left(2^{13} c_{1} b_{2} \eta i_{5}\right) \in H$, где $\eta=\operatorname{det} h_{1}$. $\operatorname{det} h_{2} \in R^{*}$, а потому и включение

$$
t_{12}\left(2^{13} c_{1} b_{2} i_{5}\right) \in H
$$

Аналогично, “транспонировав" рассуждение, получим

$$
t_{12}\left(2^{13} b_{1} c_{2} i_{5}\right) \in H
$$

В силу симметрии из (12) и (13) получаем также включения

$$
t_{12}\left(2^{13} b_{1} c_{2} i_{5}\right) \in H, \quad t_{21}\left(2^{13} c_{1} b_{2} i_{5}\right) \in H .
$$


Рассмотрев теперь вместо $h_{1}$ матрицу $t_{21}^{-1}(\beta) h_{1} t_{21}(\beta)$, где $\beta \in I$, вместо (12) получим

$$
t_{12}\left(2^{13}\left(c_{1}+\left(d_{1}-a_{1}\right) \beta-b_{1} \beta^{2}\right) b_{2} i_{5}\right) \in H .
$$

Заменив здесь $\beta$ на $-\beta$, имеем

$$
t_{12}\left(2^{13}\left(c_{1}-\left(d_{1}-a_{1}\right) \beta-b_{1} \beta^{2}\right) b_{2} i_{5}\right) \in H .
$$

Из $(15)$ и (16) получим $t_{12}\left(2^{14}\left(d_{1}-a_{1}\right) b_{2} \beta i_{5}\right) \in H$ или ввиду произвольности $\beta \in I$

$$
t_{12}\left(2^{14}\left(d_{1}-a_{1}\right) b_{2} i_{6}\right) \in H .
$$

Ввиду (14) в этих рассуждениях трансвекции $t_{12}$ можно заменить трансвекциями $t_{21}$. Поэтому

$$
t_{21}\left(2^{14}\left(d_{1}-a_{1}\right) b_{2} i_{6}\right) \in H .
$$

В силу симметрии из (17) и (18) получаем также включения

$$
t_{12}\left(2^{14} b_{1}\left(d_{2}-a_{2}\right) i_{6}\right) \in H, \quad t_{21}\left(2^{14} b_{1}\left(d_{2}-a_{2}\right) i_{6}\right) \in H .
$$

“Транспонировав" теперь эти рассуждения, вместо (17) и (18) получим

$$
t_{12}\left(2^{14}\left(d_{1}-a_{1}\right) c_{2} i_{6}\right) \in H, \quad t_{21}\left(2^{14}\left(d_{1}-a_{1}\right) c_{2} i_{6}\right) \in H,
$$

а также в силу симметрии

$$
t_{12}\left(2^{14} c_{1}\left(d_{2}-a_{2}\right) i_{6}\right) \in H, \quad t_{21}\left(2^{14} c_{1}\left(d_{2}-a_{2}\right) i_{6}\right) \in H .
$$

Далее, из (12), (15) и (17) имеем $t_{12}\left(2^{14} b_{1} b_{2} \beta^{2} i_{5}\right) \in H$ или ввиду произвольности $\beta \in I$

$$
t_{12}\left(2^{15} b_{1} b_{2} i_{7}\right) \in H .
$$

Аналогично получается включение

$$
t_{21}\left(2^{15} c_{1} c_{2} i_{7}\right) \in H .
$$

Наконец, рассмотрев вместо $h_{1}$ матрицу $t_{21}^{-1}(\beta) h_{1} t_{21}(\beta)$, где $\beta \in I$, вместо (21) получим

$$
\begin{aligned}
& t_{12}\left(2^{14}\left(c_{1}+\left(d_{1}-a_{1}\right) \beta-b_{1} \beta^{2}\right)\left(d_{2}-a_{2}\right) i_{6}\right) \in H, \\
& t_{21}\left(2^{14}\left(c_{1}+\left(d_{1}-a_{1}\right) \beta-b_{1} \beta^{2}\right)\left(d_{2}-a_{2}\right) i_{6}\right) \in H .
\end{aligned}
$$

Отсюда ввиду $(19),(21)$ и произвольности $\beta \in I$ имеем

$$
t_{12}\left(2^{14}\left(d_{1}-a_{1}\right)\left(d_{2}-a_{2}\right) i_{7}\right) \in H, \quad t_{21}\left(2^{14}\left(d_{1}-a_{1}\right)\left(d_{2}-a_{2}\right) i_{7}\right) \in H .
$$

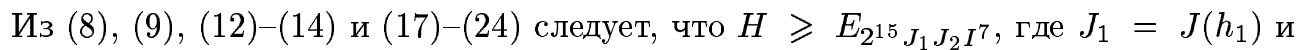
$J_{2}=J\left(h_{2}\right)$.

Ввиду произвольности матриц $h_{1}, h_{2} \in H$ отсюда получаем включение $H \geqslant E_{J}$, где $J=2^{15} J^{2}(H) I^{7}$. Далее, подгрупшы $H^{g}, g \in \mathrm{SL}_{2}(R)$, также нормализуются нормальной подгруппой $K_{I}$. Поскольку $J\left(H^{g}\right)=J(H)$, то только что доказанное включение дает $H^{g} \geqslant E_{J}$, где $J=2^{15} J^{2}(H) I^{7}$. Отсюда $H \geqslant E_{J}^{g}$ для всех $g \in \mathrm{SL}_{2}(R)$. Поэтому

$$
H \geqslant E_{J}^{\mathrm{SL}_{2}(R)}=K_{J}
$$

ввиду [1, теорема III.2]. Лемма 1 доказана. 
ЛЕмма 2. Если подгруппа $H$ группь $\mathrm{GL}_{2}(R)$ нормализуется подгруппой $K_{R}=$ $\mathrm{SL}_{2}(R)$, mo $H \geqslant K_{J}$, әде $J=2^{7} J(H)$.

ДокАЗАТЕльСтво леммы разобьем на два предложения. В них условие леммы предполагается вьполненным без оговорок.

ПРЕДЛОЖЕНИЕ 1. Ecли $t_{12}(\alpha) \in H$ или $t_{21}(\alpha) \in H$, mo $H \geqslant E_{J}$, əде $J=4 \mathrm{id}(\alpha)$.

ДокАЗАТЕЛЬСТво. Поскольку

$$
\left(e_{12}(1)+e_{21}(-1)\right) t_{12}(\alpha)\left(e_{12}(1)+e_{21}(-1)\right)^{-1}=t_{21}(-\alpha),
$$

то достаточно рассмотреть случай $t_{12}(\alpha) \in H$. Пусть $r \in R$. По определению кольца $R$ существует элемент $\varepsilon \in R$ такой, что элементы $\varepsilon, r+\varepsilon^{2}$ и $r-\varepsilon^{2}$ принадлежат $R^{*}$. Имеем

$$
4 r=\left(\frac{r+\varepsilon^{2}}{\varepsilon}\right)^{2}-\left(\frac{r-\varepsilon^{2}}{\varepsilon}\right)^{2}=\varepsilon_{1}^{2}-\varepsilon_{2}^{2},
$$

где $\varepsilon_{1}, \varepsilon_{2} \in R^{*}$. Тогда $H$ содержит матрицу

$$
t_{12}(4 r \alpha)=t_{12}\left(\varepsilon_{1}^{2} \alpha\right) t_{12}\left(-\varepsilon_{2}^{2} \alpha\right)=d^{-1}\left(\varepsilon_{1}\right) t_{12}(\alpha) d\left(\varepsilon_{1}\right) d^{-1}\left(\varepsilon_{2}\right) t_{12}(-\alpha) d\left(\varepsilon_{2}\right) .
$$

Ввиду произвольности элемента $r \in R$ и с учетом равенства в начале доказательства отсюда получим $H \geqslant E_{J}$, где $J=4 \mathrm{id}(\alpha)$.

ПРЕДЛОЖЕНИЕ 2. Имеет место соотношение $H \geqslant K_{J}$, где $J=2^{7} J(H)$.

ДокАЗАТЕЛЬСТво. Пусть $h$ - матрица из $H$ указанного вьше вида. Здесь можно считать, что $a \in R^{*}$. Действительно, если это не так, то, поскольку $\operatorname{id}(a, c)=R$, существует элемент $r \in R$ такой, что $a+r c \in R^{*}$, и мы перешли бы от $h$ к матрице $t_{12}(r) h t_{12}^{-1}(r)$ из $H$, вес которой равен $J(h)$. Далее, положив $\alpha=1$ в начале доказательства леммы 1 , вместо (1) получим

$$
t_{12}\left(2^{5} c \varepsilon_{4}\right) \in H, \quad \varepsilon_{4} \in R^{*} .
$$

Аналогично, вместо (3) имеем

$$
t_{21}\left(2^{5} b \varepsilon_{5}\right) \in H, \quad \varepsilon_{5} \in R^{*} .
$$

В силу предложения 1 из (25) и (26) теперь получим

$$
\begin{aligned}
& H \geqslant E_{J}, \quad \text { где } J=2^{7} \mathrm{id}(c), \\
& H \geqslant E_{J}, \quad \text { где } J=2^{7} \mathrm{id}(b) .
\end{aligned}
$$

Наконец, рассмотрев матрицу $t_{21}(1) h t_{21}^{-1}(1)$ с учетом $(27)$, имеем

$$
H \geqslant E_{J}, \quad \text { где } J=2^{7} \mathrm{id}(a-d) .
$$

Из (27) и (28) следует, что $H \geqslant E_{J}$, где $J=2^{7} J(h)$. Ввиду произвольности $h \in H$ отсюда имеем $H \geqslant E_{J}$, где $J=2^{7} J(H)$. Поэтому $H \geqslant E_{j}^{\mathrm{SL}_{2}(R)}=K_{J}$. Предложение 2 доказано. 
ТЕОрема 1. Пусть $R$ - $(1,6)$-полное, $(2,4)$-полное или $(3,3)$-полное кольио и $\mathrm{SL}_{2}(R) \leqslant G \leqslant \mathrm{GL}_{2}(R)$. Ecлu $H \triangleleft^{d} G, m o$

$$
K_{J f(d)} \leqslant H \leqslant Z_{J(H)}
$$

где $J=2^{8} J(H)$ и $f(d)=\left(4 \cdot 7^{d-1}-1\right) / 3$, причем при $d=1$ можно полохить $J=2^{7} J(H)$.

ДоКАЗАТЕЛЬСТВо ведется индукцией по субнормальной глубине $d$ подгрупшы $H$.

При $d=1$ подгруппа $H$ нормализуется подгруппой $K_{R}=\mathrm{SL}_{2}(R)$ и по лемме 2 $H \geqslant K_{J}$, где $J=2^{7} J(H)$.

Пусть теперь $d>1$ и утверждение теоремы выполнено для всех подгрупп $M \triangleleft^{d-1} G$. Тогда $H \triangleleft M$ для некоторой такой подгрупшы $M$ и $K_{w} \leqslant M$, где $w=\left(2^{8} J(M)\right)^{f(d-1)}$. Таким образом, $H$ нормализуется подгруппой $K_{w}$, а потому по лемме 1

$$
H \geqslant K_{2^{15} J^{2}(H) w^{7}}
$$

Так как $J(H) \subseteq J(M)$, то

$$
2^{15} J^{2}(H) w^{7} \supseteq\left(2^{8} J(H)\right)^{2}\left(2^{8} J(H)\right)^{7 f(d-1)}=\left(2^{8} J(H)\right)^{7 f(d-1)+2}=\left(2^{8} J(H)\right)^{f(d)},
$$

поэтому $H \geqslant K_{J(d)}$, где $J=2^{8} J(H)$. Включение $H \leqslant Z_{J(H)}$ очевидно. Теорема доказана.

В работе [2] аналогичное описание было получено со значительно худшей функцией $f(d)=\left(7 \cdot 13^{d-1}-1\right) / 6$, причем в предположении обратимости элемента 2 в кольце $R$.

Пользуясь случаем, отметим теперь следуюшее усиление теоремы 1 из [3]:

ТЕОрема 2. Пусть $R$ - (1, 18)-полное, $(2,10)$-полное или $(3,7)$-полное кольцо и $\mathrm{SL}_{2}(R) \leqslant G \leqslant \mathrm{GL}_{2}(R)$. Ecлu $H \triangleleft^{d} G, m o$

$$
K_{J f(d)} \leqslant H \leqslant Z_{J(H)}
$$

где $J=2^{14} J(H)$ и $f(d)=\left(5^{d}-1\right) / 4$, причем при $d=1$ можно положить $J=2^{7} J(H)$.

ДокАЗАТЕльство. Для доказательства этой теоремы в лемме 1 работы [3] идеал $I^{6}$ достаточно заменить идеалом $I^{5}$, а для этого достаточно заменить включения (13) и (14) работы [3] соответственно включениями

$$
t_{21}\left(2^{14} c i_{5}\right) \in H, \quad t_{12}\left(2^{14} b i_{5}\right) \in H .
$$

Действительно, из [3, с. 417] имеем $t_{21}\left(2^{8} b i_{3}\right) \in H$ и $t_{21}\left(2^{14} c \alpha i_{5}\right) \in H$. Отсюда $t_{21}\left(2^{14}(a(a+d)-b c) c i_{5}\right) \in H$ или, поскольку $a(a+d)-b c=\operatorname{det} h \in R^{*}, t_{21}\left(2^{14} c i_{5}\right) \in H$. Аналогично доказьвается и включение $t_{12}\left(2^{14} b i_{5}\right) \in H$. Теорема доказана с учетом работы [3]. 
Отметим, что в работе [3, теорема 2] аналогичное описание дано для случая локальных колец с той же функцией $f(d)=\left(5^{d}-1\right) / 4$.

Отметим также, что в работах [4]-[6] были получены описания субнормальных подгрупп общих линейных груп $\mathrm{GL}_{n}$ при $n \geqslant 3$ соответственно с функщиями

$$
f(d)=\frac{7^{d}-1}{6}, \quad f(d)=\frac{6^{d}-1}{5} \quad \text { и } \quad f(d)=\frac{5^{d}-1}{4} .
$$

Таким образом, в описаниях субнормальных подгрупш обших линейных групш $\mathrm{GL}_{n}$, $n \geqslant 2$, наименьшей (а значит, и наилучшей) является пока функция $f(d)=\left(5^{d}-1\right) / 4$. Интересно отметить, что она же остается пока наименьшей и в случае бесконечномерных обших линейных групп [7]. С другой стороны, известно [5], что $f(d) \geqslant 2^{d}-1$.

\section{СПИСОК ЦИТИРОВАННОЙ ЛИТЕРАТУРЫ}

[1] McDonald B.R. GL Ge $_{2}$ of rings with many units // Comm. Algebra. 1980. V. 8. № 9. P. 869-888.

[2] Тажетдинов С. Субнормальное строение двумерных линейных групп над 6-примитивньми кольцами // Матем. заметки. 1992. Т. 52. № 4. С. 99-105.

[3] Тажетдинов С. Субнормальное строение двумерных линейных групп над кольцами, близкими к полям // Алгебра и логика. 1985. Т. 24. № 4. С. 414-425.

[4] Wilson J. S. The normal and subnormal structure of general linear groups // Proc. Cambridge Phil. Soc. 1972. V. 71. № 2. P. 163-177.

[5] Vaserstein L. N. Thesubnormal structure of general linear groups // Math. Proc. Cambridge Phil. Soc. 1986. V. 99. P. 425-431.

[6] Vavilov N.A. A note on the subnormal structure of general linear groups // Math. Proc. Cambridge Phil. Soc. 1990. V. 107. P. 193-196.

[7] Arrell D. G. The subnormal subgroup structure of the infinite general linear group // Proc. Edinburgh Math. Soc. 1982. V. 25. P. 81-86. 